\title{
Controller Tuning for Integrator Plus Delay Processes.
}

\author{
B. S. Patil ${ }^{1}$, Dr. L. M. Waghmare ${ }^{2}$, Dr. M. D. Uplane ${ }^{3}$ \\ ${ }^{1}$ Instrumentation Department, AISSMS'S Polytechnic, Near RTO office, Kennedy Road, Pune-01. \\ ${ }^{2}$ Director, Shri Guru Gobind Singhji, Institute of Engineering \& Technology, Vishnupuri, Nanded. \\ ${ }^{3}$ sHOD, Professor, Instrumentation Science Department, Savitribai Phule Pune University, Pune.
}

\begin{abstract}
A design method for PID controllers based on internal model control (IMC) principles, direct synthesis method $(D S)$, stability analysis (SA) method for pure integrating process with time delay is proposed. Analytical expressions for PID controllers are derived for several common types of process models, including first order and second-order plus time delay models and an integrator plus time delay model. Here in this paper, a simple controller design rule and tuning procedure for unstable processes with delay time is discussed. Simulation examples are included to show the effectiveness of the proposed method.
\end{abstract}

Keywords: PID controller, tuning, unstable processes, internal model control (IMC), direct synthesis method $(D S)$, stability analysis $(S A)$.

Date of Submission: 02 March 2017 $\longrightarrow$ Date of Accepted: 28 March 2017

\section{INTRODUCTION}

The ubiquitous PID controller has continued to be the most extensively used process control technique for many decades. Although advanced manage techniques such as model predictive control can provide major improvement, a PID controller that is correctly planned and tune has proved to be suitable for the vast majority of industrial control loops. The enormous writing on PID controllers includes a wide variety of design and tuning methods based on dissimilar performance criteria. A simple IMC-PID controller design technique is given by [9] on the basis of the IMC principle for integrating processes with time delay. The process is mainly listening carefully on the trouble rejection, which causes the overshoot in the set point reply, and a Two Degree Of Freedom (2DOF) control organization is used to eliminate this overshoot. PID controller is designed using Maclaurin series expansion. The controller is tuned for robustness by specifying the peak of maximum sensitivity.

The design methods for PID controller are classically based on a time-domain or frequency-domain performance criterion. However, the relationships between the dynamic performance of the closed-loop system and this presentation index are not straightforward. In the direct synthesis (DS) approach 13-15 however, the manager design is based on a desired closed-loop transfer function. Then, the controller is calculated systematically so that the closed-loop set-point response matches the desired response. The obvious advantage of the direct synthesis approach is that performance requirements are built-in directly through specification of the closed-loop transfer function. One way to specify the closed-loop transfer function is to choose the closed-loop poles. This pole situation method 4, 13 can be interpreted as a special type of direct synthesis.

The SIMC method for PID manager alteration (Skogestad, 2003) has already establish wide industrial usage. The SIMC rules are logically resulting, and from a first or second order process we can simply find the pi and PID controller setting, respectively. Even though the rule was originally derived largely with ease in mind, recent studies have establish that the resulting setting are very close to optimal (Grimholt and Skogestad, 2012, 2013). For the twice integrating process, the SIMC rule gives the pid setting for the serial form.

\section{LITERATURE SURVEY}

1.1 Identification and Tuning of Integrating Processes with Dead time and Inverse Response. Author- William L. Luyben.

The classical example of an integrating method with inverse reply is level control of a boiler steam drum. The "boiler swell" difficulty can lead to a transfer function between the drum level and boiler feed water flow rate that contains a clean integrator and a positive zero, in addition to some dead time and lags. a procedure for identify the transfer-function parameters for this type of structure from step response data. A proportional integral controller tuning process is also presented. Because the process contains an integrator and the proportional-integral controller also contains an integrator, manager tuning is somewhat complex. 
1.2 PI/PID Controller Design Based on Direct Synthesis and Disturbance Rejection

Author- Dan Chen and Dale E. Seborg.

A design method for PID controller based on the straight synthesis approach and condition of the desired closedloop transfer function for disturbances is proposed. Analytical expressions for PID controllers are resulting for several common types of process models, including first order and second-order plus time delay models and an integrator plus point time delay model. Although the controllers are designed for trouble rejection, the set-point responses are regularly satisfactory and can be tuned separately via a set-point weighting factor.

1.3 PID Controllers Tuning for Improved Performance of Unstable Processes.

Author- G. M. Malwatkar, P. T. Bhosale, S. D. Nikam, L. M. Waghmare.

The design procedures for unbalanced processes involve compensation of unbalanced zeros or poles. In most of the cases PID controllers are used to control the stable processes, however less common for unbalanced processes. In this paper, a simple controller design rule and tuning procedure for unstable process with delay time is discussed. The method is developed based on a $-3 \mathrm{~dB}$, gain intersect and phase crossover frequencies with Pade estimate for the time delay. Simulation examples are included to show the efficiency of the proposed method.

1.4 Performance Enhancement in Disturbance Rejection by PID Controller in Series with Low Pass Filter. Author- Sneha S. Patil, Gajanan M. Malwatkar, Jayant V. Kulkarni.

It is based on the direct synthesis approach. Analytical expressions for the PID manager are derived for a common type of process model. Although the controller is designed for trouble rejection, the set point response is satisfactory and can be improved by adjusting a single set point weighing factor $b$. Three simulation examples show that the proposed design method results in extremely good control for process models. IAE (Internal absolute error) of the planned method is have smaller value than DS-d controller.

1.5 Design of PID Controllers for Improved Performance of Higher Order Systems.

Author- L. M. Waghmare G. M. Malwatkar.

Frequency reply based design method of PID controller is proposed for higher order (HO)/-plus delay point in time (HOPDT) systems. The HO/-PDT models are converted into real and unreal part at a incidence where a criteria similar to amplitude and phase margin is utilized to get the constraints on the parameter of the controllers. To discard sound and load disturbances, the constraint given by sensitivity and complementary sensitivity function are also considered. The proposed method has larger features for systems with high order, time delays and monotonic or oscillatory responses.

1.6 Design of Centralized PI/PID Controller: Interaction Measure Approach.

Author- G. M. Malwatkar, A. A. Khandekar, V. G. Asutkar and L. M. Waghmare.

Control system design involves input/output (IO) range, that is, decisions on the number, the place, and the type of actuators and sensors. The choice of inputs and outputs affects the presentation, complexity, and cost of the control system. In most of the multivariable systems controllers are planned by decomposing multi-loop systems into a number of corresponding single loops and design of a manager for each loop is performed. The success of controller depends on the input output pairing as it decides the structure of the controller. In this paper design rules and tuning system of the controller for multivariable process with delay point in time are discussed without decomposing multivariable system.

\section{SYSTEM METHODS}

1.7 Direct Synthesis Method Based on Set point Responses:

In the direct synthesis approach [5], an analytical expression is derived for the process model and desired closedloop transfer function. Consider a feedback control system in Fig.1.

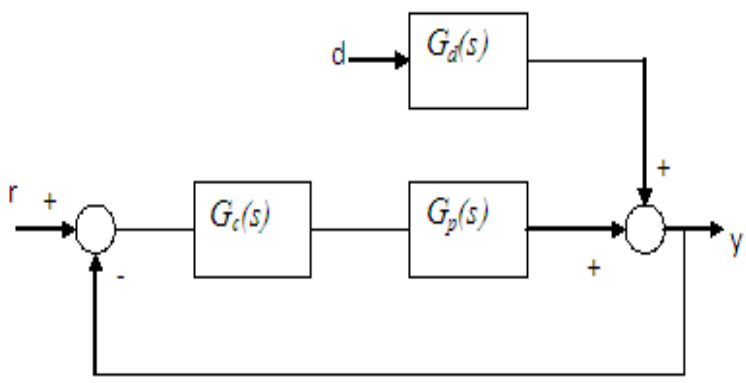

Fig.1. Feedback control system 
Assume that $\mathrm{Gp}(\mathrm{s})$ is a model of the process. The closed-loop transfer function for set point changes is derived as:

$$
\frac{y}{r}=\frac{G_{p}(s) G_{k}(s)}{1+G_{p}(s) G_{c}(s)}
$$

Rearranging the terms,

$$
G_{c}(s)=\frac{\left(\frac{y}{r}\right)}{G_{p}(s)\left[1-\left(\frac{y}{r}\right)\right] \ldots \ldots \ldots . .2}
$$

Let the desired closed-loop transfer function for set point change is specified as $(\mathrm{y} / \mathrm{r})_{d}$, and assume that process model $\mathrm{G}_{\mathrm{p}}(\mathrm{s})$ is available. Replacing the unknown $(\mathrm{y} / \mathrm{r})$ and $\mathrm{G}_{\mathrm{p}}(\mathrm{s})$ by $(\mathrm{y} / \mathrm{r})_{d}$ and $\mathrm{G}_{\mathrm{p}}(\mathrm{s})$. Respectively [1] gives.

$$
G_{c}(s)=\frac{\left(\frac{y}{r}\right)_{d}}{\tilde{G}_{p}(s)\left[1-\left(\frac{y}{r}\right)_{d}\right]}
$$

Because the individuality of $(\mathrm{y} / \mathrm{r})_{\mathrm{d}}$ have a direct impact on the resulting controller $(\mathrm{y} / \mathrm{r})_{\mathrm{d}}$ be supposed to be chosen so that the closed-loop presentation is suitable and the resulting controller is actually realizable.

1.8 IMC design method:

Lee et al. extended the IMC design approach for two degree of freedom controllers to improve disturbance performance. Their controller is a combination of two controllers, a standard IMC controller for set-point changes and a second IMC type of controller designed to shape the disturbance response. Their control system also includes a set-point filter that is specified as the inverse of the IMC controller for disturbances. This design provide a set-point reply that is equal to that for the standard IMC controller.This novel control scheme can provide improved dynamic performance over standard IMC controllers, but the design procedure is more complicated and might result in unstable controllers.

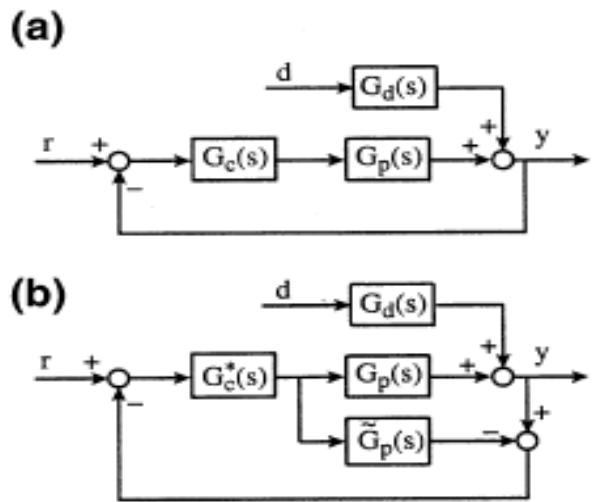

Fig.2. Feedback control strategies. (a) Classical feedback control. (b) Internal model control.

1.9 Robust stability test using Kharitonov's theorem:

A control system is supposed to be robust, if the blocked loop system is stable even when the model parameter of the actual method are dissimilar than that used for the controller design. The robust stability of a linear timeinvariant control system contain a plant which has some transfer function coefficients subject to perturbation. The set of transfer function generated by varying these coefficients in arranged ranges correspond to a box in parameter space is referred to as an interval plant. To compare the strength of the different controller design methods, the stability region in each of the model parameters for which the manager is stable is to be calculated. The stability of such system is a problem in the theory of robust control with structured perturbations. Kharitonov's theorem (Kharitonov's, 1978) that deals with the stability of an interval polynomial could be suitably global to deal with this problem.

Kharitonoves theorem is used to determine the stability of an uncertain system by major four bounding polynomials and applying Routh - Hurwitz examination to each of them. It gives a necessary and enough 
condition for all polynomials in a given family to be Hurwitz stable (Callier and Desoer, 1991) In this, the family of polynomials considered is obtain by allowing each of the polynomial coefficients to vary separately within an interval. It shows that stability of this family of polynomials can be determined by looking at the stability of four specially constructed vertex polynomial.

1.9.1 Theorem 1. (Kharitonov 1978a)

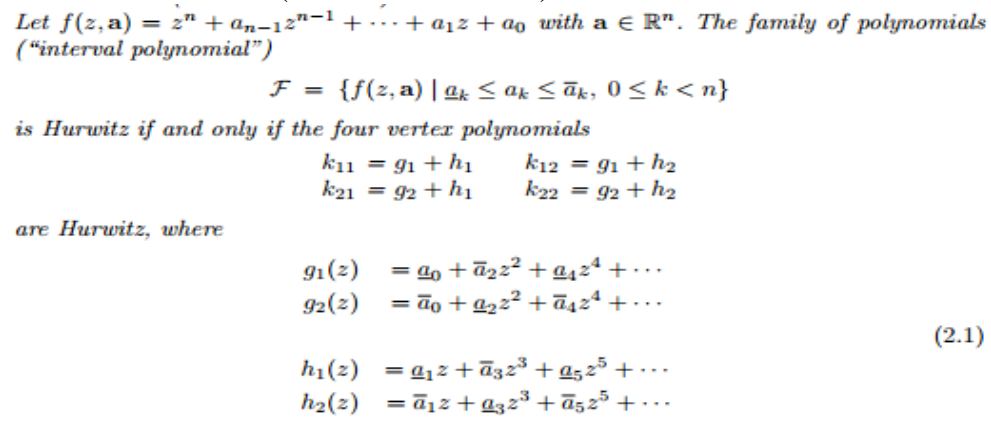

1.9.2 Algorithm:

For each of the four Kharitonov polynomial

1. Form the Hurwitz matrix.

2. Perform modified gaussian elimination $r$ closed row reduction to get.

3. Examine the diagonal elements for sign change.

4. If there is a sign change in the diagonal elements, the polynomial is not Hurwitz stable.

5. If all the four set of polynomials are Hurwitz stable, the system is proved for its robust stability. $\mathrm{H} \mathrm{n} \mathrm{H}$

6. If any one set of polynomial is not Hurwitz the system is not robustly stable.

\section{CONCLUSION}

Three methods of designing PID controllers for pure integrating system with time delay are proposed based on IMC method, stability analysis method and direct synthesis method. The performance of the proposed controllers is better than the recently reported methods. Stability region for various model parameters considering uncertainty in one parameter at a time is obtained using theorem and compared with that of the literature reported methods. The stability region for all the model parameters is comparable with that of the literature reported methods. The advantage of these methods is that the controller is PID and simple conventional feedback control structure is used.

\section{ACKNOWLEDGEMENT}

I would prefer to give thanks the researchers likewise publishers for creating their resources available. Additionally, I appreciative to commentator for their vital recommendations moreover I am conjointly grateful to reviewer for their valuable suggestions and also thank the college authorities for providing the required infrastructure and support.

\section{REFERENCES}

[1]. Chien, L. and Fruehauf, P. S. 1990. Consider IMC tuning to improve performance. Chem Eng Progr, 10: 33-41.

[2]. Fuentes, C. and Luyben, W. L. 1983. Control of high purity distillation columns. Ind Eng Chem Process Des Dev, $22: 362$.

[3]. Srividya, R. and Chidambaram, M. 1997.On line controller tuning for integrator plus delay processes. Process Contr Qual, 9: 5966.

[4]. Astrom, K. J., and Haggland, T. 1995. "PID controllers: Theory, Design and Tuning”, Instr Soc. America, North Carolina.

[5]. Yu, C. C. 1999. "Auto Tuning of PID controllers", Springer-Verlag, Berlin

[6]. Ziegler, J. G. and Nichols, N. B. 1942. Optimum settings for automatic controllers. Trans ASME, 64: 759-765.

[7]. Tyreus, B.D. and Luyben, W.L. 1992. Tuning PI controllers for integrator/ dead-time processes. Ind Eng Chem Res, $31: 2625$.

[8]. Chidambaram, M. 1994. Design of PI controller for integrator/dead-time processes. Hungar J Ind Chem, $22: 37$.

[9]. Poulin, E. and Pomerleau, A. 1999. PI settings for integrating processes based on ultimate cycle information. IEEE Trans Contr Syst Tech, 7: 509.

[10]. Luyben, W. L. 1996. Design of Proportional Integral and Derivative controllers for integrating dead-time processes. Ind Eng Chem Res, 35: 3480

[11]. Wang, L. and Cluette, W.R. 1997. Tuning PID controllers for integrating processes. IEE Proc Contr Theor Appl, $144: 385$.

[12]. Kookos, I. K., Lygros, A. I., and Arvanitis, K. G. 1999. On-line PI controller tuning for integrator / dead time processes. Eur J Contr, 5: 19.

[13]. Wang, Y.G. and Cai, W.J. 2002. Advanced proportional integral derivative tuning for integrating and unstable processes with gain and phase margin specifications. Ind Eng Chem Res, 41: 2910-2914.

[14]. Visioli, A. 2001. Optimal tuning of PID controllers for integral and unstable processes. IEE Proc Contr Theor Appl, 148: 180.

[15]. Zhang, W., Xu, X., and Sun, Y. 1999. Quantitative performance design for integrating processes with time delay. Automatica, 35: 719-723. 